\title{
artigo
}

Pires, D.J.R.; Marta, C.B.; Oliveira, E.S.; Bridi, A.C.; Franco, A.S.; Lyra da Silva, R.C.; Peregrino, A.A.F.

Avaliação de usabilidade de bomba infusora no Centro de Terapia Intensiva: contribuições para segurança do paciente

\section{Avaliação de usabilidade de bomba infusora no Centro de Terapia Intensiva: contribuilções para segurança do paciente}

\author{
Evaluation of usability of an infusion pump in the Intensive Care Center: contributions to patient safety \\ Evaluación de la usabilidad de una bomba de infusión en el Centro de Cuidados Intensivos: contribuciones a la \\ seguridad del paciente
}

\begin{abstract}
RESUMO
Objetivos: avaliar o grau de satisfação, conhecimento e a usabilidade situada da bomba infusora utilizada no Centro de Terapia Intensiva geral adulto de um hospital universitário; identificar o que sabem os profissionais de enfermagem acerca da bomba de infusão que operam; analisar os atributos de usabilidade da bomba infusora na perspectiva dos usuários; verificar o grau de satisfação do usuário como atributo de usabilidade em relação às bombas de infusão. Método: Trata-se de estudo descritivo, com abordagem quantitativa, realizado na unidade de Terapia Intensiva geral adulto de um hospital universitário do Rio de Janeiro, com 41 profissionais e residentes de enfermagem, através de um questionário. Resultado: 75,6\% conhecem a bomba infusora utilizada no setor, e a maioria a considera de fácil manuseio. 38 profissionais afirmam ter um conhecimento bom do equipamento. Conclusão: Esta pesquisa traduz a importância de avaliar em cenário real as questões relacionadas à usabilidade dos equipamentos médico-assistenciais, considerando a satisfação dos usuários que manuseiam este equipamento.
\end{abstract}

DESCRITORES: Bombas de Infusão; Enfermagem; Cuidados Críticos; Usabilidade.

\section{ABSTRACT}

Objectives: to evaluate the degree of satisfaction, knowledge and the located usability of the infusion pump used in the general adult intensive care center of a university hospital; identify what nursing professionals know about the infusion pump they operate; analyze the usability attributes of the infusion pump from the users' perspective; verify the degree of user satisfaction as a usability attribute in relation to infusion pumps. Method: This is a descriptive study, with a quantitative approach, carried out in the general adult intensive care unit of a university hospital in Rio de Janeiro, with 41 nursing professionals and residents, through a questionnaire. Result: $75.6 \%$ know the infusion pump used in the sector, and most consider it to be easy to handle. 38 professionals claim to have a good knowledge of the equipment. Conclusion: This research reflects the importance of evaluating in a real scenario the issues related to the usability of medical assistance equipment, considering the satisfaction of the users who handle this equipment.

DESCRIPTORS: Infusion Pumps; Nursing; Critical Care; Usability.

\section{RESUMEN}

Objetivos: evaluar el grado de satisfacción, conocimiento y la facilidad de uso localizada de la bomba de infusión utilizada en el centro general de cuidados intensivos para adultos de un hospital universitario; identificar lo que los profesionales de enfermería saben sobre la bomba de infusión que operan; analizar los atributos de usabilidad de la bomba de infusión desde la perspectiva de los usuarios; verificar el grado de satisfacción del usuario como un atributo de usabilidad en relación con las bombas de infusión. Método: Estudio descriptivo, con enfoque cuantitativo, realizado en la unidad general de cuidados intensivos para adultos de un hospital universitario de Río de Janeiro, con 41 profesionales de enfermería y residentes, a través de un cuestionario. Resultado: el 75,6\% conoce la bomba de infusión utilizada en el sector, y la mayoría considera que es fácil de manejar. 38 profesionales afirman tener un buen conocimiento del equipo. Conclusión: Esta investigación refleja la importancia de evaluar en un escenario real los problemas relacionados con la usabilidad del equipo de asistencia médica, considerando la satisfacción de los usuarios que manejan este equipo. este equipo.

DESCRIPTORES: Bombas de Infusión; Enfermería; Cuidados Críticos Usabilidad.

RECEBIDO EM: 08/02/2020 APROVADO EM: 10/02/2020

\section{Deywisson de Jesus Ribeiro Pires}

Enfermeiro. Especialista em Terapia Intensiva pelo Programa de Residência em enfermagem da Universidade Estadual do Rio de Janeiro - UERJ. https://orcid.org/0000-0001-5135-4931 


\section{Cristiano Bertolossi Marta}

Enfermeiro. Pós-Doutor em Enfermagem. Professor Adjunto 2 do Departamento de Fundamentos de Enfermagem da Faculdade de Enfermagem da Universidade do Estado do Rio de Janeiro. Coordenador Geral da Pós-Graduação de Enfermagem em Alta Complexidade da Universidade Veiga de Almeida. Diretor de Pesquisa da Universidade Veiga de Almeida. https:/orcid. org/0000-0002-0635-7970

\section{Elson Santos de Oliveira}

Doutorando em Enfermagem e Biociências- UNIRIO; Professor Assistente do curso de graduação de Enfermagem da Universidade VEIGA de Almeida; Enfermeiro do CTI Adulto do Instituto Nacional de Câncer. https://orcid.org/0000-0001-9377-0140

\section{Adriana Carla Bridi}

Doutorado em Enfermagem e Biociências Universidade Federal do Estado do Rio de Janeiro-Unirio. Hospital Universitário Pedro Ernesto-Universidade do Estado do Rio de Janeiro-Hupe-Uerj. https://orcid.org/0000-0003-2018-4604

\section{Andrezza Serpa Franco}

Doutorado. Universidade Federal do Estado do Rio de Janeiro. Professora assistente II Departamento Médico cirúrgico, UERJ. https://orcid.org/0000-0001-5008-1345

\section{Roberto Carlos Lyra da Silva}

Doutorado. Professor Associado da Escola de Enfermagem Alfredo Pinto. Universidade Federal do Estado do Rio de Janeiro. Líder do Laboratórios de Avalição Econômica e Tecnologias em Saúde - LAERS. https://orcid.org/0000-0003-4066-7451

\section{Antônio Augusto de Freitas Peregrino}

Professor Associado - UERJ. Doutorado saúde coletiva instituto de medicina social - UERJ. Pós doutorado em enfermagem e biociências - Unirio. https://orcid.org/0000-0002-6617-480X

\section{INTRODUÇÃO}

A aquisição tecnológica nos serviços de saúde público e privado, sobretudo para as unidades de terapia intensiva, representa um avanço no cuidado aos pacientes críticos. No entanto, diante da necessidade de melhorar a qualidade dos serviços de saúde prestados aos pacientes, os profissionais de saúde deparam-se com equipamentos médico-assistenciais cada vez mais complexos e indispensáveis à assistência.

No cenário de cuidados intensivos, para manter a vida e recuperar a saúde, além dos cuidados diretos aos pacientes, é fundamental o conhecimento teórico e prático do manuseio das máquinas para que se compreenda seu funcionamento e se interprete os dados, o que pode garantir a confiabilidade dos resultados e o direcionamento da assistência.

Sendo assim, essas tecnologias que colaboraram no tratamento e diagnóstico dos pacientes internados em uma unidade de terapia intensiva podem ser deletérias com consequências diretas na segurança do paciente, caso o usuário não esteja familiarizado com suas funcionalidades ${ }^{(1)}$.
Um bom exemplo destas tecnologias é o uso das bombas infusoras. Equipamentos essenciais em terapia intensiva para realização de medicamentos que necessitam ter sua dosagem com controle rigoroso, como aminas, sedativos, entre outros. Um desconhecimento no processo de manipulação destas tecnologias poderá levar o paciente a eventos adversos importantes.

A avaliação de tecnologia em saúde auxilia os gestores dos setores público e privado de saúde a tomar decisões racionais na escolha de quais tecnologias devem ser disponibilizadas e no planejamento da alocação de recursos financeiros em saúde, considerando desempenho, custo de manutenção, adoção desnecessária, índice de reparos, uso inadequado e obsolescência do equipamento ${ }^{(2)}$.

Os equipamentos tecnológicos estão mais presentes nos setores dos serviços de saúde e, por conseguinte, os profissionais de saúde têm que lidar com as adequações e devem se capacitar para a utilização e melhor interpretação dos resultados obtidos. Ressaltando que com a incorporação dos novos equipamentos médico-assistenciais dentro das unidades de cuidados inten- sivos, que se caracterizam por cuidados complexos, pode alterar a carga cognitiva desses profissionais durantes suas atividades, comprometendo a eficiência na realização das tarefas ${ }^{(3)}$.

Neste cenário destaca-se o uso de tecnologias nos sistemas de infusão que constantemente vem apresentando progresso, ao passo que este deve ser acompanhado com adequada usabilidade do equipamento dentro das unidades, e que possa garantir a segurança do paciente e satisfação do usuário.

Diante do exposto traçamos como questão deste estudo: A usabilidade da bomba infusora pela equipe de enfermagem da unidade de terapia intensiva reduz os erros na assistência de enfermagem?

O desfecho primário é a satisfação e conhecimento da bomba infusora pelos profissionais de enfermagem de um CTI geral adulto; e o secundário é a usabilidade da bomba infusora em um CTI geral adulto.

Assim, os objetivos deste estudo são: avaliar o grau de satisfação, conhecimento e a usabilidade da bomba infusora utilizada no Centro de Terapia Intensiva geral adulto de um hospital universitário; identificar o perfil dos profissionais de enfer- 


\section{artigo}

Pires, D.J.R.; Marta, C.B.; Oliveira, E.S.; Bridi, A.C.; Franco, A.S.; Lyra da Silva, R.C.; Peregrino, A.A.F.;

Avaliação de usabilidade de bomba infusora no Centro de Terapia Intensiva: contribuições para segurança do paciente

magem que atuam no CTI geral adulto; e verificar a satisfação e o conhecimento dos usuários em relação às bombas de infusão.

\section{METODOLOGIA}

Esta pesquisa é de natureza descritiva, com abordagem quantitativa. Com avaliação de tecnologia em saúde, tendo por escolha a usabilidade da bomba de infusão.

O cenário em que foi realizada esta pesquisa é o Centro de Terapia Intensiva Geral Adulto do Hospital Universitário Pedro Ernesto, no município do Rio de Janeiro, que apresenta 10 leitos. Nesta unidade o equipamento de bomba de infusão utilizado é o Infusomat compact B BRAUN.

Como participantes desta pesquisa, são os profissionais de enfermagem que atuam no setor do centro de terapia intensiva, incluindo os residentes de enfermagem. Tendo um total 69 usuários, sendo 12 enfermeiros, 14 residentes e 43 técnicos de enfermagem.

Esta pesquisa avaliou as seguintes variáveis: características das atividades desenvolvidas pelos usuários; marca, modelo e características técnicas da bomba de infusão; parâmetros da bomba de infusão; dificuldades encontradas pelos usuários para utilizar o EMA; satisfação do usuário em relação a bomba de infusão.

Utilizou-se a aplicação de um questionário eletrônico aos participantes, compreendendo em duas etapas: conhecer perfil dos profissionais de enfermagem que atuam no CTI geral; avaliar a satisfação e conhecimento do usuário em relação a utilização da bomba de infusão. Os dados obtidos foram categorizados por variáveis, e analisadas por estatística descritiva simples.

Atendendo as questões éticas, esta pesquisa foi submetida e aprovada pelo Comitê de Ética em Pesquisa da Universidade do Estado do Rio de Janeiro, sob parecer n. ${ }^{\circ} 3.138 .283-08$ de fevereiro de 2019.

\section{RESULTADOS E DISCUSSÃO}

Os dados foram coletados em maio a julho de 2019. A pesquisa contou com um total de 41 participantes, profissionais de enfermagem usuários da bomba de in- fusão, sendo 21 enfermeiros e 20 técnicos de enfermagem. Os dados foram obtidos em duas etapas: (1) caracterização do perfil dos usuários da bomba de infusão; (2) manuseio da bomba de infusão.

\section{Caracterização do perfil dos usuá- rios da bomba de infusão}

A descrição e caracterização do perfil dos usuários da bomba infusora que atuam no CTI geral adulto foi realizada a partir $\mathrm{da}$ identificação de características como sexo/ gênero, formação profissional, idade, anos de experiência profissional, turno de trabalho e número de vínculos empregatícios.

Quanto ao perfil dos usuários, elencamos dados referentes ao sexo/ gênero de profissionais que atuam na unidade, contando com 29 (70,7\%) profissionais do sexo feminino, fato que corrobora com o perfil global dos profissionais de enfermagem que, em sua maioria, são do sexo feminino ${ }^{(4)}$. No que refere à formação profissional, a categoria de enfermeiros que respondeu ao questionário foi em maior quantitativo que a dos técnicos de enfermagem. Este fato justifica-se pelo quantitativo maior de enfermeiros no cenário do estudo, e por se tratar de um hospital universitário, 11 (53\%) destes enfermeiros que responderam a pesquisa são residentes do programa de enfermagem em terapia intensiva.

A idade dos usuários neste estudo teve predominância entre 20 a 29 anos, e 30 a 39 anos, com idade média de 35,2 anos com idade máxima de 57 anos e mínima de 20 anos. É uma equipe de profissionais que ainda representa uma classe de trabalhadores novos, numa lógica de expectativa de vida. Se pensarmos que esta categoria, que está exposta a diversos riscos, especificamente no cuidado a pacientes intensivos, que exige do profissional um esforço físico muito intenso, além de grau de agilidade, destreza e energia. Acreditamos que esse intervalo de idade possa ser benéfico para a realização das atividades e a manutenção do padrão de qualidade da assistência prestada ${ }^{(3,5)}$.

Ao relacionar a variável idade deste usuário com a usabilidade da bomba infusora, podemos analisar a questão da manipulação de novas tecnologias, que se mostra mais eficiente quando utilizadas por profissionais mais novos, pois eles apresentam menores dificuldades para manipulá-la $a^{(3)}$.

Outro fator que pode gerar mais benefícios à assistência segura e de qualidade é o fato desses jovens profissionais trabalharem em conjunto com profissionais com mais experiência. Esse encontro possibilita uma construção coletiva de prática atual e segura baseada em tecnologia e segurança do paciente.

No que refere à atuação profissional, 21 (51,2\%) profissionais tem o tempo superior a 10 anos de experiência. Esse dado nos mostra que a equipe possui conhecimento apropriado para o manuseio do cuidado e das tecnologias presentes no setor. $\mathrm{O}$ fato que nos leva a pensar que esses profissionais podem ter utilizado inúmeras bombas infusoras de marcas e tipos diferentes, e tecnologias menos atuais que a tecnologia em avaliação. Estudo ressalta que os profissionais com mais experiência se apresentam mais bem preparados diante diversas situações já vivenciadas, envolvendo a assistência a pacientes críticos em unidades intensivas e, a partir das experiências, atuam de modo mais resolutivo dentro do cenário de cuidados críticos ${ }^{(6)}$.

De todos os usuários participantes, 31 $(75,6 \%)$ conhecem outras marcas e modelos de bomba infusora, destes 10 (32,3\%) consideram que outros modelos são de mais fácil manipulação do que a disponível na unidade. Está relacionado com a satisfação do usuário da tecnologia prejudicada, pois a maioria deles sente dificuldade no manuseio da tecnologia.

Os resultados mostram que a unidade apresenta uma equipe composta por profissionais com experiência, um fator importante quando se trata de cuidados intensivos, pois há especificidades na assistência exigindo de seus trabalhadores $\mathrm{o}$ alto padrão de conhecimento técnico e científico, além de diversas outras competências para o trabalho em equipe ${ }^{(1,3)}$.

A variável de distribuição dos profissionais a partir do turno de trabalho mostrou que a maioria, 25 profissionais 
trabalham no serviço diurno e noturno (61\%), e 16 (39\%) trabalham apenas no serviço diurno. Em relação ao número de vínculos empregatícios, em sua grande maioria atuam em 1 (51,2\%) ou 2 (46,3\%) vínculos empregatícios.

Em estudo acerca da caracterização de eventos adversos nas unidades intensivas, aponta que os eventos adversos com maior incidência ocorrem no turno da manhã, e que podem estar relacionados a maior quantidade de procedimentos(7). Ressalta-se que os profissionais que atuam no período noturno estão relacionados com quantitativo maior de horas de trabalho em comparação a outros turnos que podem levar ao estresse e fadiga dos profissionais; e ainda a subnotificação de eventos pode ser um fator para avaliação.

\section{Manuseio da bomba de infusão}

Além da caracterização, o estudo apresenta variáveis que investigam e identificam as que estão relacionadas ao manuseio da bomba de infusão, tais como: conhecimento e a familiaridade que o usuário tem para identificar problemas e/ou ter ações resolutivas; a questão de treinamento em serviço para o manuseio da bomba; o grau de usabilidade e satisfação pelos usuários.

$\mathrm{Na}$ análise da variável de satisfação do usuário a respeito da usabilidade da bomba infusora, no que se refere a conhecer a marca e modelo da bomba disponível na unidade, $85,4 \%(\mathrm{n}=35)$ sabem informar os dados da bomba infusora, destes, $43,9 \%$ eram enfermeiros e $41,5 \%$, técnicos de enfermagem.

Entendendo a familiaridade como capacidade que o usuário tem de compreender e operar todas as funcionalidades sem dificuldades, $85,7 \%(\mathrm{n}=18)$ dos enfermeiros e $100 \%(n=20)$ de técnicos de enfermagem afirmam ter familiaridade com a bomba infusora. Tal resultado retrata o perfil de uma equipe resolutiva atuante na unidade, o que pode minimizar a ocorrência de eventos adversos relacionados ao uso do equipamento.

A bomba infusora é destacada como uma tecnologia que representa segurança dentre os equipamentos médico-assisten- ciais, no entanto, a utilização deste equipamento não é garantia para a não ocorrência de evento adverso, uma vez que a tecnologia depende da intervenção humana na sua programação ${ }^{(8)}$.

A Figura 1 demonstra o grau de conhecimento dos recursos da bomba de infusão pelos usuários. Os dados apresentam que os sujeitos do estudo afirmam ter conhecimento dos recursos disponíveis. Esse aspecto é positivo para a minimização dos riscos envolvendo a utilização do equipamento. Esse conhecimento pode estar relacionado ao fato de termos muitos profissionais com mais de 10 anos de experiência, fato que aumenta a probabilidade de já ter entrado em contato com tal tecnologia.

Estudo aponta a importância da implementação da sistematização e de protocolos de rotinas para proporcionar uma assistência de qualidade, primando pela segurança do paciente ${ }^{(9)}$. Ao passo que a implementação de rotinas está associada à consciência e questões cognitivas desse profissional usuário que manuseia a bomba infusora, pautadas para uma adesão responsável e a utilização de recursos disponíveis.

A usabilidade da bomba infusora pelos usuários foi avaliada por algumas questôes que tratam diretamente como os mesmos

Figura 1. Nível de conhecimento dos recursos da bomba infusora pelos usuários. Rio de Janeiro, RJ, Brasil, 2019.

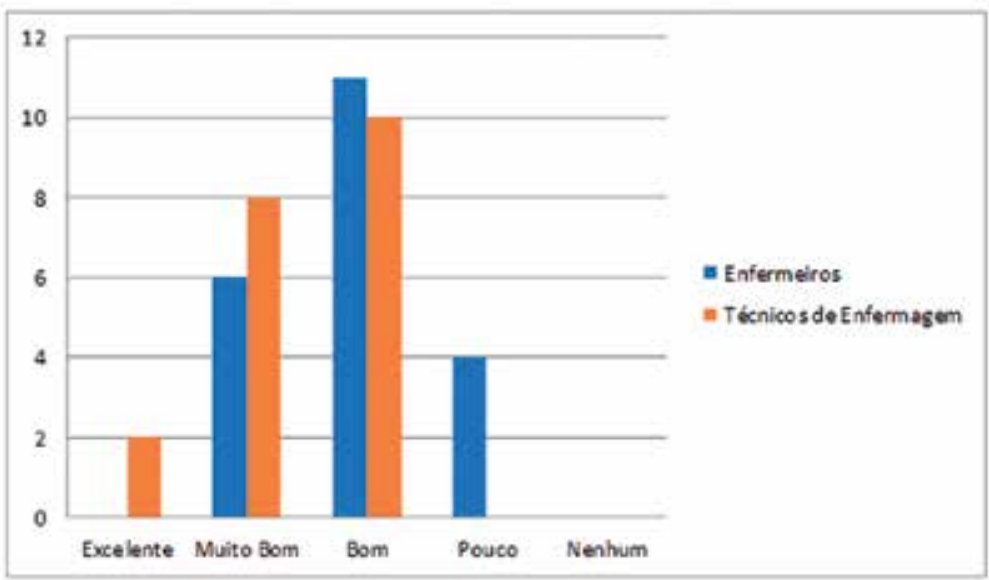

percebem essa tecnologia e se é uma tecnologia que contribui positivamente para a assistência. No item que mede o quanto os usuários sabem operar, considerando que o profissional saiba compreender as mensagens e informações fornecidas no display ou dos alarmes luminosos e sonoros emitidos pela bomba adequadamente, dos 41 profissionais, 4 enfermeiros e 1 técnico de enfermagem referem dificuldade para operar a bomba infusora.

Quando os usuários são questionados se sabem interpretar as mensagens no display ou aos alarmes sonoros e/ou luminosos fornecidas pela bomba, apenas 1 enfermeiro apontou não saber identificar e compreender de modo a tomar decisão resolutiva.

Em relação às possíveis dificuldades relatadas pelos usuários deste estudo no que se relaciona com a não familiaridade com o dispositivo, referem a tela do display e a configuração. Esse resultado corrobora com o estudo que aponta como principais dificuldades das funcionalidades: a tela do display, configurações, compreender as mensagens do alarme e display ${ }^{(3)}$.

No que se refere aos alarmes da bomba infusora, 10 enfermeiros $(47,6 \%)$ e 14 técnicos de enfermagem (70\%) consideram que os alarmes das bombas são fundamentais para a prática. E, em seguida, os enfer- 


\section{artigo}

Pires, D.J.R.; Marta, C.B.; Oliveira, E.S.; Bridi, A.C.; Franco, A.S.; Lyra da Silva, R.C.; Peregrino, A.A.F.

Avaliação de usabilidade de bomba infusora no Centro de Terapia Intensiva: contribuições para segurança do paciente

meiros $(\mathrm{n}=7)$ consideram ainda que a não há valorização do alarme pela equipe.

Num estudo desenvolvido, que refere aos alarmes pela bomba infusora e à caracterização dos alarmes, houve mais evidência do alarme de "fim de infusão" (41,7\%), fato que pode ser considerado risco para o paciente; em segunda evidência foi "manipulação de bombas infusoras" (29,2\%) que gera ruídos e alarmes que podem ter implicações negativas para os pacientes e aos próprios profissionais que manuseiam este equipamento ${ }^{(10)}$.

Os alarmes gerados pela bomba infusora podem ser fator de risco para o profissional e dificultador para a recuperação dos pacientes. Estudos apontam que os ruídos contribuem para sintomas de estresse como fadiga pessoal, problemas de concentração e tensão ${ }^{(1,10)}$.

Quando os usuários relatam a sua experiência com o uso da bomba infusora, houve $1(2,4 \%)$ usuário enfermeiro que refere uma experiência negativa com o equipamento, justificando que os alarmes estressam a equipe.

Apesar de apenas 1 usuário (2,4\%) indicar experiência negativa com o manuseio do equipamento, houve quatro usuários $(9,7 \%)$ que consideraram não estarem satisfeitos com a bomba disponível na unidade.

Para a avaliação da satisfação dos usuários da bomba infusora na unidade de cuidados intensivos, foi aplicada uma escala numérica de 0 a 10 , na qual 0 representa totalmente insatisfeito e 10, totalmente satisfeito. Percebe-se, pelos dados produzidos, que os usuários indicam um bom nível de satisfação, tendo uma média de 7,97.

A usabilidade da bomba infusora nas unidades de terapias intensivas, a expressiva falta de adesão aos treinamentos e a capacidade do profissional ignorar ou, até mesmo, burlar os recursos disponíveis pelo equipamento médico-assistencial são os principais obstáculos para a redução da fadiga de alarmes e, consequentemen- te, para a prestação de uma assistência de enfermagem de qualidade, que prioriza a segurança do paciente.

\section{CONCLUSÃO}

Esta pesquisa traduz a importância de avaliar em cenário real as questões relacionadas à usabilidade dos equipamentos médico-assistenciais, considerando a satisfação dos usuários que manuseiam este equipamento. Compreende-se que os objetivos propostos foram alcançados através de um método que permitiu a avaliação da usabilidade da bomba infusora e o grau de satisfação pelos profissionais de enfermagem.

Os resultados encontrados relacionados à usabilidade da bomba infusora se mostraram significativamente positivos; ao passo que a satisfação do usuário com o equipamento também apresenta um resultado significativo, configurando pela possibilidade de manuseio desta bomba infusora, em comparação a outros modelos.

\section{REFERÊNCIAS}

1. Vasconcellos LG. Avaliação da usabilidade situada de bombas de infusão em uma unidade de cuidados intensivos. 2015. $111 \mathrm{f}$. Dissertação (Mestrado em Enfermagem). Escola de Enfermagem Alfredo Pinto, Universidade Federal do Estado do Rio de Janeiro - UNIRIO, Rio de Janeiro, 2015.

2. Ministério da Saúde, Secretaria de Ciência, Tecnologia e Insumos Estratégicos (BR). Diretrizes metodológicas: elaboração de estudos para avaliação de equipamentos médicos-assistenciais. Brasília: Ministério da Saúde, 2013.

3. Caldeira FN. Avaliação situada de usabilidade de bombas infusoras em uma unidade de terapia intensiva. Rio de Janeiro, 2017. 84f. Dissertação (Mestrado em Enfermagem). Escola de Enfermagem Alfredo Pinto, Universidade Federal do Estado do Rio de Janeiro - UNIRIO, Rio de Janeiro, 2016.

4. Silva JDF. O enfermeiro no exercício de uma profissão predominantemente feminina: uma revisão integrativa. 46f. Monografia (Graduação em Enfermagem). Universidade Federal do Maranhão. São Luís, 2018.

5. Oliveira ES. Avaliação situada de usabilidade de bombas de infusão: um estudo sobre a satisfação de usuários e as implicações para segurança do paciente em terapia de infusão intravenosa contínua. Rio de Janeiro, 2015. 122f. Dissertação (Mestrado em Enfermagem). Escola de Enfermagem Alfredo Pinto, Universidade Federal do Estado do Rio de Janeiro - UNIRIO, Rio de Janeiro, 2015.
6. Santos F. O tempo estímulo-resposta na predisposição a fadiga de alarmes de ventiladores mecânicos: implicações para a segurança do paciente. Dissertação (Mestrado em Enfermagem) - Rio de Janeiro. Universidade Federal do Estado do Rio de Janeiro, 2013

7. Souza RF, Alves AS, Alencar IGM. Eventos adversos na unidade de terapia intensiva. Rev. enferm. UFPE online [Internet]. 2018 Jan. [citado 28 set. 2018]; 12(1):19-27. Disponivel em: https:// doi.org/10.5205/1981-8963-v12i01a25205p19-27-2018.

8. Moreira APA, Escudeiro CL, Christovam BP, Silvino ZR, de Carvalho MF, da Silva RCL. Uso de tecnologias na terapia intravenosa: contribuições para uma prática mais segura. Rev. bras. Enferm [Internet]. 2017 [citado 28 set. 2018]; 70(3):595-601. Disponivel em: http://dx.doi.org/10.1590/0034-7167-2016-0216.

9. Moreira APA. Incorporação tecnológica no contexto da saúde: uma análise de custo efetividade das bombas de infusão na insulinoterapia intravenosa. Rio de Janeiro, 2017. 104f. Tese (Doutorado em Enfermagem). Escola de Enfermagem Alfredo Pinto, Universidade Federal do Estado do Rio de Janeiro - UNIRIO, Rio de Janeiro, 2017.

10. Franco AS, Luna AA, Camerini FG, Henrique DM, Almeida LF, da Silva RCL. Segurança na utilização de bombas infusoras: análise dos alarmes. Rev. enferm. UFPE on-line [Internet]. $2018 \mathrm{Mai}$ [citado 20 jul 2019]; 12(5): 1331-7. Disponivel em: https://doi.org/10.5205/1981-8963-v12i5a231286p1331-1337-2018. 